\title{
La thérapie génique ouvre une nouvelle ère
}

\author{
Tuy Nga Brignol, Sylvie Marion, Emmanuelle Salort-Campana, Gisèle Bonne
}

Année après année, les techniques de thérapie génique s'affinent et se diversifient avec déjà des résultats très encourageants au niveau clinique. La preuve lors d'une session présidée par Serge Braun et Giuseppe Ronzitti au congrès Myology 2019 qui s'est déroulé à Bordeaux du 25 au 28 mars.

\begin{abstract}
Deux nouvelles voies prometteuses dans la dystrophie musculaire de Duchenne

Différents traitements basés sur l'utilisation de matériel génétique sont en développement dans la dystrophie musculaire de Duchenne (DMD). La plupart repose sur la délivrance, par un virus adéno-associé (AAV), d'un gène thérapeutique codant une microdystrophine.

Lors du congrès Myology 2019, Kevin Flanigan du Center for Gene Therapy (Columbus, États-Unis) a présenté deux alternatives utilisant des AAV. Les deux approches peuvent être considérées comme complémentaires des thérapies par la microdystrophine, pour différents sous-groupes de patients.
\end{abstract}

Thérapie génique de substitution par surexpression du gène GALGT2

Le gène GALGT2 code la bêta1,4-N-acétylgalactosaminyltransférase ( $\beta-1,4-G a l N A c$ transférase) ou GALGT2. La protéine GALGT2 endogène est généralement exprimée dans le muscle au niveau de la synapse neuromusculaire et de la jonction myotendineuse, là où l'utrophine remplace la dystrophine dans un complexe protéique associé à l'utrophine. La surexpression de GALGT2 entraîne l'expression de l'utrophine dans l'ensemble de la fibre musculaire, ainsi qu'une régulation positive d'autres protéines associées à la stabilisation de la membrane de la cellule musculaire.

Tuy Nga Brignol Sylvie Marion AFM-Téléthon, Évry, France

Emmanuelle

Salort-Campana

Centre de référence

des maladies

neuromusculaires

et de la SLA, Hôpital

de la Timone. Marseille,

France

Gisèle Bonne

Sorbonne Université,

Inserm UMRS 974

Institut de Myologie,

GH Pitié-Salpêtrière,

Paris, France

Contact

tnbrignol@afm-telethon.fr
La surexpression de GALGT2 dans les muscles squelettiques de souris $m d x$ inhibe le développement de la dystrophie musculaire. Chez des souris $m d x$, cette surexpression permet une conservation significative de la force mesurée lors de contractions excentriques et empêche la perte de force des muscles non dystrophiques. Dans un protocole de thérapie génique intravasculaire, une comparaison de la surexpression de GALGT2 et de la microdystrophine a montré que GALGT2 est aussi efficace pour prévenir la perte de force lors de contractions excentriques [1]. Dans un modèle de primate non humain, l'isolement transitoire du flux sanguin des membres postérieurs à l'aide d'un cathéter à ballonnet a permis de concentrer le vecteur dans les muscles des jambes. Une dose bilatérale de $2,5 \times 10^{13}$ génomes de vecteur $(\mathrm{vg}) / \mathrm{kg} / \mathrm{membre}$ s'est montrée suffisante pour provoquer une glycosylation induite par GALGT2 dans $10 \%$ à $60 \%$ des myofibres squelettiques de l'ensemble des muscles des jambes traitées. Avec la même dose, le génome des vecteurs par microgramme d'ADN génomique a augmenté en moyenne de 6 à 19 fois pour l'ensemble du membre. Une dose unilatérale de $6 \times 10^{13} \mathrm{vg} / \mathrm{kg} /$ membre administré à l'aide d'un cathéter à ballonet a montré une augmentation de $12 \pm 3$ fois dans les muscles du membre traité par rapport à ceux du membre controlatéral n'ayant reçu le vecteur qu'après libération dans la circulation systémique à partir du membre traité. La variabilité de la biodistribution des AAV entre différents segments du même muscle a été de $125 \% \pm 18 \%$ pour une dose donnée, tandis que la variabilité entre le même muscle des deux côtés pour une dose de traitement donnée a été de $45 \% \pm 7 \%$.

Ces expériences démontrent que le traitement des muscles de la jambe avec rAAVrh74.MCK.GALGT2 peut être réalisé en toute sécurité en utilisant une technique de perfusion isolée du membre, mais la variabilité intra- et inter-musculaire reste importante [2].

Cette approche fait l'objet d'un essai clinique ouvert de phase I/Ila (NCT03333590; https://clinicaltrials.gov/ct2/show/NCT03333590) à doses croissantes aux États-Unis. Son objectif est d'évaluer l'innocuité (signes cliniques, dosages biologiques sériques et urinaires, réponse immunologique à rAAVrh74 et à GALGT2) et l'efficacité (test de marche de 6 minutes ou 6MWT, testing musculaire des muscles des membres inférieurs) de l'administration intravasculaire isolée de rAAVrh74.MCK. GALGT2 chez six patients âgés de plus de quatre ans atteints de DMD.

Le vecteur est administré, via l'artère fémorale, aux muscles des membres inférieurs. Une procédure de perfusion intravasculaire modifiée est utilisée pour 
administrer séquentiellement le vecteur. L'essai, qui a débuté en novembre 2017, a déjà inclus deux patients âgés de 8,4 ans et 6,9 ans. Présentés par Kevin Flanigan lors de Myology 2019, les résultats préliminaires montrent que le traitement est bien toléré et qu'il existe, à ce jour, une preuve d'efficacité limitée. Il n'y a pas de déclin évident au niveau de la force ou de la fonction motrice. La fin de l'essai est prévue pour novembre 2020.

Expression de la dystrophine par des petits ARN nucléaires (ARNs) U7 non codants

La seconde approche consiste à fournir plusieurs copies de petits ARN nucléaires (ARNs) U7 non codants avec des séquences ciblant les sites donneur et accepteur de l'épissage de l'exon 2, ce qui entraîne de facto l'exclusion de l'exon 2 de l'ARNm du gène de la dystrophine.

Chez les patients présentant une duplication de l'exon 2 (la duplication d'exon simple la plus fréquente), il en résulte un transcrit de type sauvage (si un exon est exclu) ou un transcript sans exon 2 , auquel cas la traduction à partir d'un site d'entrée de ribosome interne en aval est activé. L'isoforme qui en résulte est hautement fonctionnelle malgré l'absence de la première moitié du domaine de liaison à l'actine 1 (ABD1), comme le montre son expression chez des patients atteints de dystrophie musculaire de Becker. Testée chez la souris, cette stratégie entraine bien une expression prolongée de dystrophine. Un essai clinique serait prévu pour 2019.

\section{Une isoforme fonctionnelle \\ de la dystrophine traduite par un exon DMD 5 IRES}

La traduction d'un exon DMD 5 IRES donne une isoforme fonctionnelle de la dystrophine qui atténue la dystrophinopathie chez l'homme et la souris. La plupart des mutations qui tronquent le cadre de lecture du gène DMD entraînent une perte d'expression de la dystrophine et conduisent à la dystrophie musculaire de Duchenne. Cependant, il a été démontré que la gravité de la maladie peut être améliorée par l'initiation alternative de la traduction commençant dans l'exon 6 de DMD, ce qui conduit à l'expression d'une dystrophine $\mathrm{N}$-tronquée hautement fonctionnelle. L'équipe de Kevin Flanigan a démontré que cette isoforme entraîne l'utilisation d'un site d'entrée interne du ribosome (IRES pour internal ribosome entry site) dans l'exon 5 qui est inductible par les glucocorticoïdes. Ils ont confirmé l'activité de l'IRES par séquençage des peptides et par profilage du ribosome dans le muscle de patients présentant des symptômes mineurs de DMD malgré la présence de mutations tronquantes. Ils ont généré un cadre de lecture tronqué en amont de l'IRES par saut d'exon, ce qui a conduit à la synthèse d'une isoforme N-tronquée fonctionnelle dans des lignées cellulaires humaines dérivées et dans un nouveau modèle murin DMD. Par rapport aux souris témoins, l'expression du muscle soumis aux contractions, et protégé par l'isoforme tronquée a pu être corrigée. Ces résultats soutiennent une approche thérapeutique possible pour les patients présentant des mutations dans les exons situés dans la région 5' du gène DMD [3].

\section{Trp3X : un allèle fondateur du gène DMD}

Une mutation récurrente non-sens de l'exon $1 \mathrm{du}$ gène $D M D$, p.Trp3X (c.9G>A) a été constatée pour la première fois chez un patient décédé à 77 ans et présentant un début de faiblesse musculaire proximale à l'âge de 20 ans. Il a conservé la marche jusqu'à l'âge de 62 ans. Cette mutation nonsens DMD p.Trp3X est associée à un effet fondateur dans les familles nord-américaines atteintes de formes bénignes de dystrophie musculaire de Becker.

Six autres familles non apparentées portant une mutation p.Trp3X ont ensuite été identifiées, cinq en Amérique du Nord et une en Italie. Dans six de ces sept familles, le cas-index a présenté dans l'enfance des taux élevés de créatine kinase détectés de manière fortuite, ou dans le cadre de bilan de crampes, avec ou sans épisode de rhabdomyolyse. L'analyse génétique par génotypage de SNP haute densité a montré que les six familles nord-américaines partagent un haplotype de 3,7 Mbp entourant l'allèle p.Trp3X, ce qui signifie qu'il s'agit d'une mutation fondatrice chez ces individus. La taille de l'haplotype fondateur et la structure de segments partagés d'un génome à l'autre suggèrent que l'âge minimal de cette mutation est supérieur à six générations.

La découverte de cette première mutation fondatrice du gène $D M D$, associée à un phénotype bénin de dystrophie musculaire de Becker, suggère que la prévalence des mutations hypomorphiques ${ }^{1}$ de la dystrophine devrait être réexaminée à l'aide d'une analyse génomique améliorée [4].

\section{Après la SMA, des stratégies basées} sur l'AAV pour les formes familiales de SLA La thérapie génique est également d'actualité pour les formes génétiques, familiales, de sclérose latérale amyotrophique (SLA). Cette maladie se traduit par une dégénérescence des motoneurones, comme l'amyotrophie spinale proximale liée à SMN1 (SMA). Dans la SMA comme dans la SLA, il faut 
utiliser un vecteur viral capable d'atteindre le système nerveux central, où se trouvent les motoneurones.

Les formes familiales de la SLA représentent $10 \%$ des cas de SLA. Dans $20 \%$ des cas, elles sont liées au gène SOD1 (superoxyde dismutase 1) et à une accumulation de la protéine SOD1, pour laquelle un modèle de souris existe. Plusieurs approches ont été testées pour cibler SOD1.

Après avoir développé un AAV de type 9 pour la SMA, l'équipe de Martine Barkats (Institut de Myologie, Paris) a mis au point une stratégie moléculaire innovante visant à induire une réduction substantielle, généralisée et durable du taux de SOD1 humain mutant (hSOD1) chez des souris Sod $1^{\mathrm{G} 93 \mathrm{~A}}$. Lors de Myology 2019, Maria-Grazia Biferi (Institut de Myologie) a rapporté que cette stratégie s'avère efficace.

L'administration d'AAV10-U7-hSOD à des souris Sod $1^{\mathrm{G} 93 \mathrm{~A}}$, injectées à la naissance ou à l'âge de 50 jours, a allongé leur survie respectivement de $92 \%$ et $58 \%$. Ce traitement a aussi empêché la perte de poids et le déclin de la fonction neuromusculaire. Les résultats de l'injection intra-cérébroventriculaire (ICV) surpassent ceux de l'injection intraveineuse (IV). L'injection combinée ICV-IV n'améliore pas les résultats par rapport à ICV seule [5].

En parallèle, elle développe une approche comparable dans une autre forme familiale de SLA, liée cette fois au gène C9ORF72.

L'équipe s'attache désormais à optimiser cette thérapie dans la perspective d'une application à l'homme par une approche "Effacer/Remplacer" (Erase/Replace) pour SOD1-ALS.

\section{Un rêve devenu réalité dans la myopathie myotubulaire}

Les développements de thérapie génique sont plus avancés pour la myopathie myotubulaire, une maladie neuromusculaire congénitale liée à l'X et due à des mutations du gène MTM1 codant la myotubularine. La maladie entraine le plus souvent un décès dans la petite enfance, avant l'âge de deux ans dans plus de $50 \%$ des cas. L'équipe d'Ana BujBello (Généthon, Évry) a développé une biothérapie basée sur l'administration intraveineuse d'un AVV recombinant de type 8 pour apporter le gène MTM1 à l'ensemble des muscles. Les résultats spectaculaires chez des animaux modèles de la maladie [6] ont permis le lancement, en septembre 2017 aux États-Unis puis en Europe, d'un essai clinique international de phase I/II (étude ASPIRO) mené par Audentes Therapeutics en partenariat avec Généthon (NCT03199469; https://clinicaltrials.gov/ct2/show/NCT03199469).

Cette étude à doses ascendantes et à traitement différé vise à évaluer l'innocuité et l'efficacité préliminaire du produit AT132, un vecteur AAV8 contenant une copie fonctionnelle du gène humain MTM1 (hMTM1). Le produit est administré une seule fois par voie intraveineuse. Il est prévu d'inclure 12 participants âgés de moins de cinq ans. Des résultats préliminaires ont été dévoilés par Ana Buj-Bello. À ce stade, huit garçons atteints de myopathie myotubulaire ont été inclus: sept dans la cohorte $1\left(1,0 \times 10^{14} \mathrm{vg} / \mathrm{kg}\right.$ d'AT132), un dans la cohorte $2\left(3.0 \times 10^{14} \mathrm{vg} / \mathrm{kg}\right.$ d'AT132). À un an de leur inclusion, les huit participants sont améliorés sur le plan moteur (échelle de mesure de la fonction motrice CHOP INTEND) et respiratoire [pression inspiratoire maximale (PImax)]. Tous contrôlent leur tête et tiennent assis sans aide pendant plus de 5 secondes. Sur le plan respiratoire, la PImax est améliorée chez tous les patients dès quelques semaines après l'administration, et pour certains elle atteint des valeurs normales. Trois enfants n'ont déjà plus besoin d'assistance respiratoire. La biopsie musculaire a montré une amélioration significative de l'aspect histologique des fibres musculaires à la $24^{e}$ semaine post-injection. Le produit est généralement bien toléré. La dose prévue pour la cohorte 3 est égale à $5.0 \times 10^{14} \mathrm{vg} / \mathrm{kg}$ d'AT132.

Gene therapy opens a new era

\section{LIENS D'INTÉRÊT}

Les auteurs déclarent n'avoir aucun lien d'intérêt concernant les données publiées dans cet article.

\section{RÉFÉRENCES}

1. Martin PT, Xu R, Rodino-Klapac LR, et al. Overexpression of Galgt2 in skeletal muscle prevents injury resulting from eccentric contractions in both $\mathrm{mdx}$ and wild-type mice. Am J Physiol Cell Physiol 2009; 296 : C476-88.

2. Xu R, Jia Y, Zyomunt DA, et al. An isolated limb infusion method allows for broad distribution of rAAVrh74.MCK.GALGT2 to leg skeletal muscles in the Rhesus macaque. Mol Ther Methods Clin Dev 2018; 10 : 89-104.

3. Wein N, Vulin A, Falzarano MS, et al. Translation from a DMD exon 5 IRES results in a functional dystrophin isoform that attenuates dystrophinopathy in humans and mice. Nat Med 2014 ; 20 : 992-1000.

4. Flanigan KM, Dunn DM, von Niederhausern A, et al. DMD Trp3X nonsense mutation associated with a founder effect in North American families with mild Becker muscular dystrophy. Neuromuscul Disord 2009; 19 : 743-8.

5. Biferi MG, Cohen-Tannoudji M, Cappelletto A, et al. A new AAV10-U7-mediated gene therapy prolongs survival and restores function in an ALS mouse model. Mol Ther 2017 ; 25 : 2038-52. 6. Goddard MA, Burlingame E, Beggs AH, et al. Gait characteristics in a canine model of X-linked myotubular myopathy. J Neurol Sci 2014 ; 346 : 221-6. 